\title{
Effects of phylogeny and prey type on fatty acid calibration coefficients in three pinniped species: implications for the QFASA dietary quantification technique
}

\author{
David A. S. Rosen ${ }^{1, *}$, Dominic J. Tollit ${ }^{1,2}$ \\ ${ }^{1}$ Marine Mammal Research Unit, University of British Columbia, 2202 Main Mall, Vancouver, British Columbia V6T 1Z4, \\ Canada \\ ${ }^{2}$ Present address: SMRU Ltd, 3069 6th Avenue West, Vancouver V6K 1X4, Canada
}

\begin{abstract}
Quantitative fatty acid signature analysis (QFASA) has been proposed as a technique for determining the long-term diet of animals. The method compares the fatty acid (FA) profiles of predators and potential prey items to estimate relative prey intake. We tested the assumptions of a key step in QFASA, the correction of predator FA signatures for metabolic processes through sets of calibration coefficients (CCs). We conducted long-term controlled feeding studies with captive Steller sea lions consuming herring and eulachon and northern fur seals consuming herring. We compared the results with data from harbour seals eating herring to evaluate the effects of phylogeny and prey type on individual CCs. Even within the limited extended dietary FA subset recommended for use by other researchers, we found that at least $41 \%$ of the CCs differed by family (otariid vs. phocid seals) and 58\% differed by predator species (sea lion vs. fur seal), suggesting that CCs may be highly species-specific. We also found that $64 \%$ of the CCs differed by prey type (sea lions consuming herring vs. eulachon), which raises some fundamental implementation issues. We also found significant differences in diet predictions when the herring- and eulachon-derived sets of CCs were applied to an actual multi-species diet. CCs are presently used as a simple mathematical attempt to describe potentially complex biochemistry. The results of this study raise questions regarding the validity of using CCs derived from an alternative predator species, and highlight some fundamental issues regarding QFASA methodology that need to be addressed through further controlled studies.
\end{abstract}

KEY WORDS: Fatty acids - Quantitative fatty acid signature analysis - QFASA · Pinnipeds · Otariid · Diet

\section{INTRODUCTION}

Accurate determination of the diet of marine mammals is essential for assessing ecological interactions and potential effects on prey populations (Bowen 1997, Kenney et al. 1997, Trites et al. 1997, DeMaster et al. 2001, Bearzi et al. 2010). Traditional measures of diet determination, i.e. stomach contents and scat analysis, suffer from systematic biases and numerous logistical constraints, including the fact that they are only able to discern the immediate (24 to $48 \mathrm{~h}$ ) dietary history of an individual (reviewed by Tollit et al. 2010).

In recent years, there has been a move towards developing more temporally robust methods for determining the diets of marine predators. The 2 most established methods are stable isotope analysis and fatty acid signature analysis (FASA), which have been used to differentiate trophic interactions and implied dietary differences between different groups 
of predators on the basis of their chemical composition (Hobson 1999, Dalsgaard et al. 2003). Both of these techniques are based on the assumption that the chemical composition of a predator is heavily influenced by the chemical signature of their prey (and in the case of stable isotopes, also their environment), and that the chemical signature of specific tissues within the predator will reflect long-term diet on a potential scale from weeks to months to years. FASA examines the fatty acid (FA) composition of the predator to infer what types of prey the individual has eaten. In the specific case of using FASA with marine mammals, FAs of prey are gradually incorporated into the hypodermal blubber layer of the individual predator as triglycerides (Kirsch et al. 2000, Cooper et al. 2005), creating an integrated FA signature (or profile).

Quantitative fatty acid signature analysis (QFASA) (Iverson et al. 2004) is a first-generation statistical tool designed to provide more information than FASA by quantifying the relative abundance of specific prey items rather than just identifying what types of prey are consumed. Briefly, QFASA compares the FA profile of the predator to a 'library' of potential prey, each with a distinct FA profile. A mathematical model chooses the optimal proportion of prey that provides the 'best fit' (using Kulback-Liebler distances) to the predator's signature. QFASA has been applied to evaluate the prey of several marine homeotherms (Beck et al. 2007, Iverson et al. 2007, Thiemann et al. 2007, Tucker et al. 2009, Meynier et al. 2010).

Marine mammals may make excellent subjects for this technique given their extensive hypodermal blubber layer that can be readily sampled. In validation studies conducted to date, QFASA has been reported to be able to provide reasonably accurate estimates of the main diet items in relatively simple diets (1 to 2 prey items) in captive marine mammals and is able to detect short- and long-term switches to a new prey species (e.g. Iverson et al. 2004, Cooper et al. 2005, Nordstrom et al. 2008). However, accurately determining more complex multi-species diets has proven more difficult for a variety of reasons (Tollit et al. 2010).

It is acknowledged that the FA profile of the prey and predator will not be exact matches due to various physiological processes, including preferential accumulation or mobilization and transformation of FAs into different forms (Grahl-Nielsen et al. 2003, Andersen et al. 2004, Iverson et al. 2004). A key premise of using FA signatures to estimate diet is based on the principle that identifiable long-chain FAs from prey species are incorporated into the blubber of the predator in a predictable fashion (Iverson 1993).
Hence, one of the first, critical steps in the mathematical process of QFASA is to adjust the FA signature of the predator to account for the effects of these physiological processes. Specifically, the QFASA process requires adjusting or weighting the FA signatures of the predator by applying experimentally derived calibration coefficients (CCs). These CCs are obtained from empirical studies comparing the prey and predator FA signatures after consumption of a constant, known diet for an extended period of time (i.e. sufficient for complete FA turnover).

To date, few CCs have been determined experimentally, and scientists employing QFASA have often had to use those derived for other predator species. Currently, only a handful of CCs exist for phocid seals ('true seals') and no CCs are available for any otariid species (fur seals and sea lions). It is unclear how interchangeable these CCs might be, and whether specific calibrations might be required at the level of family or even species. There are also questions of whether CCs derived from a given prey species can be applied to an animal consuming prey with a wholly different FA profile. Potentially, specific CCs would need to be developed using experimental diets similar to those consumed in the wild. These issues are considered absolutely critical for the successful implementation of QFASA.

In this study, we present CCs experimentally derived from 2 otariid species (northern fur seals Callorhinus ursinus, NFS; and Steller sea lions Eumetopias jubatus, SSL), consuming identical, single-species diets. Developing methods to determine the prey of these species is of particular importance, given their recent population declines (National Marine Fisheries Service 2007, 2008). By combining the results of our controlled feeding experiments with data from a previously published study of a phocid species (Nordstrom et al. 2008), we specifically examined the effects of predator family and species on CCs and discuss their potential effects on the QFASA technique. For 1 otariid species, we also compared results of CCs experimentally derived from an alternative prey species to determine the potential effect of prey composition on CCs.

\section{MATERIALS AND METHODS}

\section{Sample analysis}

Blubber biopsies were obtained from 4 groups of captive individuals that were fed single-species diets for extensive periods. All individuals were fed on an 
ad libitum basis within training restrictions, were healthy, and were not pregnant, lactating or chemically or surgically neutered. Ten biopsies were obtained from subadult female SSL that had been fed Pacific herring Clupea pallasii for $>200$ d. Additional biopsies were obtained $>1$ yr later from 2 of the same SSL after consuming eulachon Thaleichthys pacificus for $>165$ d. Samples were also obtained from 6 young female captive NFS ( 18 mo old) that had consumed herring for at least $1 \mathrm{yr}$. Both the SSL and NFS had been captured as pups, housed at the Vancouver Aquarium (British Columbia, Canada), and had been trained to take part in a broad conservation research programme. Data from biopsies previously obtained from 4 captive subadult harbour seals Phoca vitulina richardsii ( $\mathrm{PV}$; Nordstrom et al. 2008) were reanalyzed to incorporate the same statistical techniques employed with the rest of the samples in this study as detailed below. These PV (3 male, 1 female) were $>7 \mathrm{yr}$ old and had originally been brought into the Aquarium from either a rehabilitation programme or a university research facility. They had eaten herring from a single lot exclusively for $>1 \mathrm{yr}$ prior to their biopsies. The time periods during which the diets were consumed in all of these studies ( $>5 \mathrm{mo}$ ) are considered to represent sufficient time for fairly complete FA turnover (e.g. Iverson et al. 2004, Hoberecht 2006, Nordstrom et al. 2008).

The biopsies were obtained in a similar fashion for all samples. All sampling was conducted under the authority of individual animal care permits issued by the University of British Columbia. Samples were obtained from the individuals while under gas anaesthesia (isoflurane) administered under veterinary supervision. The area to be biopsied (flank region between the spine and lateral line, $\sim 5$ to $10 \mathrm{~cm}$ above the pelvic girdle) was shaved and sanitized with isopropyl alcohol and iodine. A veterinarian made a $\sim 3 \mathrm{~cm}$ surgical incision with a size 11 scalpel to allow for the insertion of a 6 to $8 \mathrm{~mm}$ biopsy punch. The punch was inserted and twisted with gentle pressure applied to make a plug in the blubber, aiming to reach the level of the fascia (blubber/muscle interface). On removal with angled tweezers, the blubber sample was immediately wrapped in aluminum foil. Depending on the veterinary decision, 1 or 2 stitches might be used to close the incision. The biopsy site was monitored twice a day for infection. A 3 d course of oral antibiotics was administered for a few initial biopsies, but as no infections were observed over the course of the initial biopsies, their prophylactic use was discontinued. All biopsy sites healed within 2 to 3 wk without notable infections occurring.
Within 5 min of extraction, the blubber sample was blotted dry of excess blood, laid on foil and any muscle/fascia removed. The sample was weighed (to within $100 \mu \mathrm{g}$ ) and stored in glass vials (with Teflonlined lids) containing chloroform with $0.01 \%$ BHT and stored frozen at $-30^{\circ} \mathrm{C}$ to limit further oxidation. Individual whole prey ( $\mathrm{n}=20$ to 30 species $^{-1}$; Table 1 ) were randomly sub-sampled during the course of each trial and stored in airtight plastic bags frozen at $-20^{\circ} \mathrm{C}$ prior to analysis. Each prey sample was individually homogenized and analyzed. Total wet weight lipid content of herring $(9.9 \pm 2.8 \%$ wet weight) and eulachon $(10.2 \pm 1.8 \%)$ were similar across SSL studies.

Details of the FA extraction and quantification process can be found in Budge et al. (2002) and Iverson et al. (2004). Each set of FA signatures of blubber and matched prey were undertaken at the same laboratory, although different phases of the studies used different labs. Samples were processed in laboratories at the following institutions: SSL and PV consuming herring at the Department of Biology, Dalhousie University (Halifax, Nova Scotia, Canada); SSL consuming eulachon at the University of Alaska, Anchorage (USA); NFS consuming herring at the Food Science Program, Dalhousie University. A past inter-laboratory study of quality assurance and quality control for lipid extraction and gas chromatographic analysis for FAs (Kennish et al. 2006) has highlighted complete consistency with NIST standards in all major FAs (although not all of the laboratories used in this study were included in the original comparison). However, the limit of detection levels of 7 trace $(0.02$ to $0.4 \%$ of total signature) FAs (ai-15:0, 16:1n-5, 16:3n-6, 16:3n-1, 18:3n-1, 20:2n-9, 22:2n-6) clearly appeared to be different across laboratories, with the University of Alaska having lower detection limits for all of these FAs (see 'Results').

\section{Calibration coefficient calculation and analysis}

There are far more discernible FAs in marine lipids than are proposed for use in QFASA. Many FAs in marine ecosystems appear in quantities too small to be reliably analyzed (Kennish et al. 2006), while others are of little value for predicting diet due to predator metabolism (Iverson 1993). The FA profile produced from FA methyl ester analysis was reduced to those 66 originally selected by Iverson et al. (2004), not including 24:1n-9 (nervonic acid), which caused difficulties in their study due to a lack of predictive power (Table 1). The proportional occurrences of the 
Table 1. Fatty acid content of different prey types used in the studies, specifically herring consumed by northern fur seals (NFS HG), Steller sea lions (SSL HG) and harbour seals (PV HG), as well as eulachon fed to Steller sea lions (SSL EUL). Mean (SD) values of all prey samples are expressed as the proportion of identified fatty acids normalized to total $100 \%$ for each individual prey sample. BDL: designates when proportions were below the detection limits of the individual laboratories for all samples. Fatty acids belonging to the 'extended dietary FA' (EDFA) data subset as defined by Iverson et al. (2004) are listed first (marked with ' $\mathrm{X}$ '). 'Biopsy samples' refers to the number of individual blubber samples subsequently used for calculating calibration coefficients after consuming pure diets for the minimum feeding period

\begin{tabular}{|c|c|c|c|c|c|}
\hline Sample type & EDFA & NFS HG & SSL HG & SSL EUL & PV HG \\
\hline Prey samples (n) & & 20 & 30 & 20 & 23 \\
\hline Biopsy samples (n) & & 6 & 10 & 2 & 4 \\
\hline Feeding period (d) & & $>365$ & $>200$ & $>165$ & $>200$ \\
\hline $14: 0$ & $\mathrm{X}$ & $4.03(0.39)$ & $4.58(0.73)$ & $6.25(0.67)$ & $4.96(0.49)$ \\
\hline $16: 0$ & $\mathrm{X}$ & $20.02(1.53)$ & $19.65(1.47)$ & $15.54(1.04)$ & $19.25(1.72)$ \\
\hline $16: 1 n-7$ & $\mathrm{X}$ & $6.66(0.69)$ & $6.39(0.77)$ & $6.89(1.09)$ & $6.69(0.49)$ \\
\hline $16: 2 n-6$ & $\mathrm{X}$ & $0.085(0.012)$ & $0.077(0.024)$ & $0.0037(0.0116)$ & $0.096(0.016)$ \\
\hline $16: 2 n-4$ & $\mathrm{X}$ & $0.67(0.07)$ & $0.13(0.04)$ & $0.33(0.04)$ & $0.13(0.03)$ \\
\hline $16: 3 n-6$ & $\mathrm{X}$ & BDL & $0.60(0.18)$ & BDL & $0.76(0.12)$ \\
\hline $17: 0$ & $\mathrm{X}$ & $0.18(0.03)$ & $0.35(0.37)$ & $0.063(0.023)$ & $0.96(0.33)$ \\
\hline $16: 3 n-4$ & $\mathrm{X}$ & $0.49(0.07)$ & $0.41(0.15)$ & $0.093(0.014)$ & $0.55(0.12)$ \\
\hline $16: 3 n-1$ & $\mathrm{X}$ & BDL & $0.0031(0.0043)$ & BDL & BDL \\
\hline $16: 4 n-3$ & $\mathrm{X}$ & $0.14(0.02)$ & $0.13(0.03)$ & BDL & $0.13(0.02)$ \\
\hline $16: 4 n-1$ & $\mathrm{X}$ & $0.49(0.09)$ & $0.62(0.28)$ & $0.030(0.062)$ & $0.89(0.34)$ \\
\hline $18: 0$ & $\mathrm{X}$ & $2.88(0.30)$ & $2.52(0.35)$ & $2.45(0.38)$ & $2.29(0.28)$ \\
\hline $18: 1 n-9$ & $\mathrm{X}$ & $20.95(3.12)$ & $22.01(5.58)$ & $38.09(3.52)$ & $17.43(3.07)$ \\
\hline $18: 1 n-7$ & $\mathrm{X}$ & $4.62(0.51)$ & $3.99(0.43)$ & $7.24(0.65)$ & $3.95(0.41)$ \\
\hline $18: 2 n-6$ & $\mathrm{X}$ & $1.00(0.21)$ & $0.88(0.13)$ & $0.64(0.14)$ & $0.84(0.16)$ \\
\hline $18: 2 n-4$ & $\mathrm{X}$ & $0.16(0.03)$ & $0.12(0.03)$ & $0.049(0.040)$ & $0.14(0.03)$ \\
\hline $18: 3 n-6$ & $\mathrm{X}$ & $0.11(0.02)$ & $0.11(0.02)$ & $0.058(0.033)$ & $0.12(0.02)$ \\
\hline $18: 3 n-4$ & $\mathrm{X}$ & $0.12(0.01)$ & $0.065(0.020)$ & $0.087(0.102)$ & $0.084(0.008)$ \\
\hline $18: 3 n-3$ & $\mathrm{X}$ & $0.60(0.27)$ & $0.47(0.14)$ & $0.16(0.07)$ & $0.46(0.23)$ \\
\hline $18: 3 n-1$ & $\mathrm{X}$ & $0.037(0.008)$ & $0.044(0.022)$ & BDL & $0.056(0.014)$ \\
\hline $18: 4 n-3$ & $\mathrm{X}$ & $1.21(0.46)$ & $1.15(0.37)$ & $0.42(0.24)$ & $1.31(0.46)$ \\
\hline $18: 4 n-1$ & $\mathrm{X}$ & $0.11(0.03)$ & $0.085(0.035)$ & BDL & $0.12(0.03)$ \\
\hline 20:1n-11 & $\mathrm{X}$ & $1.50(1.11)$ & $1.37(1.27)$ & $2.62(0.82)$ & $1.51(0.81)$ \\
\hline $20: 1 n-9$ & $\mathrm{X}$ & $2.13(1.27)$ & $3.83(1.86)$ & $1.71(1.58)$ & $5.23(2.04)$ \\
\hline $20: 1 n-7$ & $\mathrm{X}$ & $0.32(0.04)$ & $0.24(0.04)$ & $0.23(0.04)$ & $0.28(0.03)$ \\
\hline $20: 2 n-6$ & $\mathrm{X}$ & $0.15(0.03)$ & $0.16(0.03)$ & $0.040(0.028)$ & $0.15(0.02)$ \\
\hline $20: 3 n-6$ & $\mathrm{X}$ & $0.068(0.010)$ & $0.067(0.013)$ & $0.023(0.025)$ & $0.073(0.012)$ \\
\hline $20: 4 n-6$ & $\mathrm{X}$ & $0.86(0.10)$ & $0.67(0.12)$ & $0.37(0.09)$ & $0.63(0.10)$ \\
\hline $20: 3 n-3$ & $\mathrm{X}$ & $0.068(0.017)$ & $0.051(0.013)$ & BDL & $0.045(0.013)$ \\
\hline $20: 4 n-3$ & $\mathrm{X}$ & $0.31(0.06)$ & $0.31(0.05)$ & $0.040(0.072)$ & $0.32(0.05)$ \\
\hline $20: 5 n-3$ & $\mathrm{X}$ & $10.45(0.99)$ & $9.85(1.65)$ & $3.13(1.40)$ & $10.67(1.65)$ \\
\hline $22: 1 n-11$ & $\mathrm{X}$ & $3.87(2.88)$ & $5.38(2.67)$ & $4.06(1.32)$ & $7.08(2.68)$ \\
\hline $22: 1 n-9$ & $\mathrm{X}$ & $0.40(0.13)$ & $0.36(0.13)$ & $0.15(0.17)$ & $0.45(0.15)$ \\
\hline $22: 1 n-7$ & $\mathrm{X}$ & $0.20(0.04)$ & $0.17(0.04)$ & BDL & $0.20(0.04)$ \\
\hline $22: 2 n-6$ & $\mathrm{X}$ & $0.014(0.005)$ & $0.0057(0.0061)$ & BDL & $0.0083(0.0087)$ \\
\hline $21: 5 n-3$ & $\mathrm{X}$ & $0.37(0.05)$ & $0.32(0.07)$ & $\mathrm{BDL}$ & $0.38(0.06)$ \\
\hline $22: 4 n-6$ & $\mathrm{X}$ & $0.082(0.011)$ & $0.028(0.022)$ & BDL & $0.035(0.044)$ \\
\hline $22: 5 n-6$ & $\mathrm{X}$ & $0.15(0.03)$ & $0.11(0.02)$ & $\mathrm{BDL}$ & $0.11(0.03)$ \\
\hline $22: 4 n-3$ & $\mathrm{X}$ & $0.041(0.014)$ & $0.035(0.012)$ & BDL & $0.039(0.013)$ \\
\hline $22: 5 n-3$ & $\mathrm{X}$ & $0.90(0.13)$ & $0.86(0.13)$ & $0.15(0.25)$ & $0.85(0.11)$ \\
\hline $22: 6 n-3$ & $\mathrm{X}$ & $10.23(1.17)$ & $8.91(1.48)$ & $5.88(1.95)$ & $7.62(1.12)$ \\
\hline $12: 0$ & & $0.089(0.008)$ & $0.090(0.012)$ & $0.075(0.013)$ & $0.084(0.010)$ \\
\hline $13: 0$ & & $0.023(0.005)$ & $0.023(0.005)$ & $0.0060(0.0099)$ & $0.025(0.006)$ \\
\hline i-14:0 & & $0.023(0.006)$ & $0.020(0.007)$ & $0.013(0.012)$ & $0.021(0.006)$ \\
\hline $14: 1 \mathrm{n}-9$ & & $0.15(0.05)$ & $0.17(0.06)$ & $0.013(0.016)$ & $0.20(0.05)$ \\
\hline $14: 1 \mathrm{n}-7$ & & $0.027(0.001)$ & $0.024(0.006)$ & $0.032(0.021)$ & $0.024(0.006)$ \\
\hline $14: 1 n-5$ & & $0.081(0.011)$ & $0.080(0.012)$ & $0.14(0.02)$ & $0.079(0.011)$ \\
\hline i-15:0 & & $0.14(0.025)$ & $0.14(0.03)$ & $0.0067(0.0167)$ & $0.15(0.03)$ \\
\hline ai-15:0 & & $0.047(0.012)$ & $0.041(0.009)$ & $\mathrm{BDL}$ & $0.044(0.011)$ \\
\hline $15: 0$ & & $0.33(0.05)$ & $0.31(0.05)$ & $0.21(0.03)$ & $0.33(0.06)$ \\
\hline $15: 1 \mathrm{n}-8$ & & $0.011(0.005)$ & $0.010(0.004)$ & BDL & $0.011(0.002)$ \\
\hline
\end{tabular}


Table 1 (continued)

\begin{tabular}{|c|c|c|c|c|c|}
\hline Sample type & EDFA & NFS HG & SSL HG & SSL EUL & PV HG \\
\hline $15: 1 n-6$ & & $0.0049(0.0054)$ & $0.0042(0.0050)$ & BDL & $0.0087(0.0027)$ \\
\hline i-16:0 & & $0.080(0.013)$ & $0.074(0.017)$ & $0.12(0.02)$ & $0.084(0.016)$ \\
\hline $16: 1 n-11$ & & $0.37(0.06)$ & $0.33(0.08)$ & $0.18(0.12)$ & $0.38(0.08)$ \\
\hline $16: 1 n-9$ & & $0.17(0.02)$ & $0.16(0.03)$ & $0.47(0.21)$ & $0.14(0.03)$ \\
\hline 7Me16.0 & & BDL & $0.19(0.06)$ & $0.15(0.06)$ & $0.21(0.05)$ \\
\hline $16: 1 n-5$ & & $0.24(0.05)$ & $0.037(0.016)$ & $0.21(0.06)$ & $0.040(0.011)$ \\
\hline $\mathrm{i}-17: 0$ & & $0.27(0.09)$ & $0.12(0.02)$ & $0.091(0.143)$ & $0.13(0.02)$ \\
\hline $17: 1$ & & $0.30(0.05)$ & $0.26(0.04)$ & $0.28(0.07)$ & $0.25(0.05)$ \\
\hline $18: 1 n-13$ & & $0.037(0.017)$ & $0.052(0.020)$ & BDL & $0.063(0.012)$ \\
\hline $18: 1 n-11$ & & $0.21(0.05)$ & $0.18(0.07)$ & $0.87(0.30)$ & $0.22(0.07)$ \\
\hline $18: 1 n-5$ & & $0.40(0.09)$ & $0.30(0.09)$ & $0.018(0.045)$ & $0.29(0.07)$ \\
\hline $18: 2 \mathrm{~d} 5,11$ & & $0.062(0.022)$ & $0.038(0.016)$ & $0.11(0.19)$ & $0.035(0.015)$ \\
\hline $18: 2 n-7$ & & $0.063(0.008)$ & $0.042(0.012)$ & $0.073(0.038)$ & $0.047(0.008)$ \\
\hline $20: 0$ & & $0.25(0.04)$ & $0.18(0.04)$ & $0.15(0.03)$ & $0.18(0.02)$ \\
\hline $20: 2 n-9$ & & $0.0011(0.0004)$ & BDL & BDL & $0.061(0.017)$ \\
\hline
\end{tabular}

complete set of 66 FAs by mass were renormalized to $100 \%$ in both prey and predator samples. The relative contribution of each FA by mass as a proportion of the complete 66 FA set is hereafter referred to as $\%$ FA.

Initial CCs were calculated by dividing the \%FA in the blubber sample for each FA by the appropriate $\%$ FA present in each fish sample separately. This resulted in a number of CCs for each FA equal to the number of fish samples.

This operation could lead to problems when \%FAs of some FAs were present in the fish or the blubber sample below minimum detection thresholds, resulting in either a division by 0 or a $\mathrm{CC}$ of 0 . The former produces a mathematical error, while the latter provides an inaccurate representation of the true physiological processes. In some fields of study, this issue is resolved by substituting results below the detection threshold with a value of one-half of the known detection threshold (Gibbons 1995). However, we found that this procedure not only resulted in highly questionable 'new' CCs but also, in some cases, grossly altered the results of the more reliably measured values. Therefore, we chose to simply eliminate data where \%FAs were below the detection limit from further consideration.

The distribution of the resulting initial CCs for each FA (1 complete set of fish samples for each predator) were log-transformed and then analyzed for statistical outliers (see trimmed mean explanation in Iverson et al. 2004). In practice, $<5 \%$ of the data were removed, since outliers were usually the result of an unusual \%FA of a single FA in only 1 or 2 fish samples. This process was repeated separately for each FA to yield a single mean $\mathrm{CC}$ for each FA for each individual blubber sample. The individual mean CCs for each FA from each animal were then combined to yield a new mean CC (and associated standard deviation, SD) for each prey and predator species (Table 2). Therefore, our final sample sizes (and related measures of variance) were set by the number of predator blubber samples and not the number of prey analyzed.

To test whether the mean CCs for a given FA were significantly different due to predator or prey species, a series of analyses of variance (ANOVAs) were applied individually to the CCs of each FA, with the means from each individual blubber sample comprising the data points within each of the 4 groups: SSL consuming herring, SSL consuming eulachon, PV consuming herring and NFS consuming herring. Comparisons between groups where none of the data were above the detection threshold were not included in this analysis (as noted in Table 2). The data were log-transformed to yield a more normal distribution. Given the large number of individual comparisons (up to 64 depending on specific comparisons), the significant alpha level was adjusted to 0.0008 for any single ANOVA so that the total chance of type I error was maintained at 0.05. Tukey post-hoc comparisons between means were employed when overall differences were significant to determine specific differences between groups. To examine the extent to which physiological processes alter the relative occurrence of FAs, we used a 2-tailed $t$-test to examine whether each mean $\mathrm{CC}$ was significantly different from 1.0 (unity between predator and prey).

Not all 66 FAs were identified as useful and of dietary origin in the original QFASA model (Iverson et al. 2004). Therefore, we repeated some of our sta- 
Table 2. Calibration coefficients (CCs) for 66 fatty acids (FAs) derived from Steller sea lions consuming herring (SSL HG) and eulachon (SSL EUL), and harbour seals (PV HG) and northern fur seals (NFS HG) consuming herring. Different subsets of FAs used primarily for QFASA analysis are noted: the 'extended dietary FA' ('E') and the 'dietary FA' subset ('D'; Iverson et al. 2004). Mean (SD) calibration coefficients are given. Mean values given in square brackets were not used in further statistical comparisons since all or all but one of the individual samples yielded a CC of zero. A series of analyses of variance (ANOVAs) were applied individually to the CCs of each FA, with the means from each individual comprising the data points within each of the 4 experimental groups. Results of ANOVAs testing overall significance are shown, and superscripts (a-d) on the means differentiate significantly different average values. These are summarized in columns denoting significant differences (designated by ' $\mathrm{X}$ ') attributable to family (PV = Phocidae, SSL and NFS = Otariidae; (X) denotes a difference between PV and either SSL or NFS but not both), species (Spp.; SSL vs. NFS) or prey type (SSL HG vs. SSL EUL). na: sets of data where comparisons could not be made. A superscript ' 1 ' on the SD denotes that the mean value is not statistically different from 1.0; BDL: below detection limit

\begin{tabular}{|c|c|c|c|c|c|c|c|c|c|c|c|}
\hline FA & Subset & NFS HG & SSL HG & SSL EUL & PV HG & Family & Spp. & Prey & $F$ & $\mathrm{df}$ & $\mathrm{p}$ \\
\hline $14: 0$ & E & $0.999^{\mathrm{c}}\left(0.016^{1}\right)$ & $0.869^{\mathrm{b}}(0.026)$ & $0.759^{\mathrm{a}, \mathrm{b}}\left(0.025^{1}\right)$ & $0.588^{\mathrm{a}}(0.014)$ & $\mathrm{X}$ & $\mathrm{X}$ & & 42.96 & 3,18 & $<0.0001$ \\
\hline $16: 0$ & $\mathrm{E}$ & $0.804^{\mathrm{c}}(0.013)$ & $0.612^{\mathrm{b}}(0.015)$ & $0.646^{\mathrm{b}}\left(0.031^{1}\right)$ & $0.479^{\mathrm{a}}(0.016)$ & $\mathrm{X}$ & $\mathrm{X}$ & & 49.12 & 3,18 & $<0.0001$ \\
\hline $16: 1 n-7$ & $\mathrm{E}$ & $0.981^{\mathrm{a}}\left(0.074^{1}\right)$ & $1.396^{\mathrm{b}}(0.077)$ & $1.074^{\mathrm{a}, \mathrm{b}}\left(0.007^{1}\right)$ & $2.377^{\mathrm{c}}(0.071)$ & $\mathrm{X}$ & $\mathrm{X}$ & & 26.87 & 3,18 & $<0.0001$ \\
\hline $16: 2 n-6$ & $\mathrm{E}, \mathrm{D}$ & $0.662^{\mathrm{b}}(0.012)$ & $0.584^{\mathrm{b}}(0.037)$ & $0.197^{\mathrm{a}}\left(0.197^{1}\right)$ & $0.745^{\mathrm{b}}(0.013)$ & & & $\mathrm{X}$ & 12.46 & 3,18 & 0.0001 \\
\hline $16: 2 n-4$ & $\mathrm{E}, \mathrm{D}$ & $0.758(0.018)$ & $0.785(0.041)$ & $0.792(0.003)$ & $0.954\left(0.037^{1}\right)$ & & & & & & 0.0403 \\
\hline $16: 3 n-6$ & $\mathrm{E}, \mathrm{D}$ & BDL & $0.607(0.036)$ & BDL & $0.759(0.052)$ & & na & na & & & 0.0439 \\
\hline $17: 0$ & $\mathrm{E}$ & $1.232^{\mathrm{C}}(0.031)$ & $0.566^{\mathrm{b}}(0.102)$ & $1.666^{\mathrm{c}}\left(0.144^{1}\right)$ & $0.100^{\mathrm{a}}(0.004)$ & $\mathrm{X}$ & $\mathrm{X}$ & $\mathrm{X}$ & 18.97 & 3,18 & $<0.0001$ \\
\hline $16: 3 n-4$ & $\mathrm{E}, \mathrm{D}$ & $0.608(0.010)$ & $0.558(0.117)$ & $0.867\left(0.027^{1}\right)$ & $0.552(0.039)$ & & & & & & 0.3031 \\
\hline $16: 3 n-1$ & $\mathrm{E}, \mathrm{D}$ & BDL & $0.933\left(0.035^{1}\right)$ & BDL & BDL & na & na & na & na & na & na \\
\hline $16: 4 n-3$ & $\mathrm{E}, \mathrm{D}$ & $0.955(0.009)$ & [0.219] & $\mathrm{BDL}$ & $0.896(0.013)$ & & na & na & & & 0.0047 \\
\hline $16: 4 n-1$ & $\mathrm{E}, \mathrm{D}$ & $0.551^{\mathrm{b}}(0.015)$ & $0.245^{\mathrm{a}}(0.016)$ & {$[0.360]$} & $0.434^{\mathrm{b}}(0.044)$ & $(\mathrm{X})$ & $\mathrm{X}$ & na & 38.74 & 2,17 & $<0.0001$ \\
\hline $18: 0$ & $\mathrm{E}$ & $1.023^{\mathrm{C}}\left(0.059^{1}\right)$ & $0.771^{\mathrm{b}}(0.042)$ & $0.703^{b}\left(0.047^{1}\right)$ & $0.456^{\mathrm{a}}(0.027)$ & $\mathrm{X}$ & $\mathrm{X}$ & & 23.19 & 3,18 & $<0.0001$ \\
\hline $18: 1 n-9$ & $\mathrm{E}$ & $1.395^{\mathrm{b}}(0.022)$ & $1.616^{\mathrm{b}, \mathrm{c}}(0.085)$ & $1.019^{\mathrm{a}}\left(0.006^{1}\right)$ & $1.921^{\mathrm{c}}(0.035)$ & $(\mathrm{X})$ & & $\mathrm{X}$ & 14.42 & $\begin{array}{l}3,10 \\
3,18\end{array}$ & $<0.0001$ \\
\hline $18: 1 n-7$ & $\mathrm{E}$ & $1.008^{\mathrm{b}}\left(0.008^{1}\right)$ & $1.153^{\mathrm{c}}(0.012)$ & $0.853^{\mathrm{a}}\left(0.014^{1}\right)$ & $1.028^{\mathrm{b}}\left(0.023^{1}\right)$ & (X) & $\mathrm{X}$ & $\mathrm{X}$ & 60.88 & 3,18 & $<0.0001$ \\
\hline $18: 2 n-6$ & $\mathrm{E}, \mathrm{D}$ & $1.215^{\mathrm{a}}(0.008)$ & $1.409^{\mathrm{b}}(0.046)$ & $1.710^{\mathrm{c}}(0.029)$ & $1.332^{\mathrm{a}, \mathrm{b}}(0.003)$ & & $\mathrm{X}$ & $\mathrm{X}$ & 12.34 & 3,18 & $<0.0001$ \\
\hline $18: 2 n-4$ & E,D & $0.887(0.021)$ & $0.840(0.037)$ & $1.109\left(0.147^{1}\right)$ & $0.691(0.018)$ & & & & & & 0.0011 \\
\hline $18: 3 n-6$ & E,D & $0.892^{\mathrm{b}}(0.007)$ & $0.668^{\mathrm{a}}(0.031)$ & $1.258^{\mathrm{C}}(0.016)$ & $0.681^{\mathrm{a}}(0.012)$ & $(\mathrm{X})$ & $\mathrm{X}$ & $\mathrm{X}$ & 27.60 & 3,18 & $<0.0001$ \\
\hline $18: 3 n-4$ & $\mathrm{E}, \mathrm{D}$ & $1.920^{\mathrm{b}}(0.068)$ & $2.442^{\mathrm{C}}(0.147)$ & $1.135^{\mathrm{a}}(0.007)$ & $2.136^{\mathrm{b}, \mathrm{c}}(0.041)$ & & $\mathrm{X}$ & $\mathrm{X}$ & 16.21 & 3,18 & $<0.0001$ \\
\hline $18: 3 n-3$ & $\mathrm{E}, \mathrm{D}$ & $1.116^{\mathrm{b}}(0.029)$ & $0.803^{\mathrm{a}}(0.033)$ & $2.537^{\mathrm{C}}(0.011)$ & $1.141^{\mathrm{b}}\left(0.060^{1}\right)$ & $(\mathrm{X})$ & $\mathrm{X}$ & $\mathrm{X}$ & 64.32 & 3,18 & $<0.0001$ \\
\hline $18: 3 n-1$ & E,D & $0.454^{\mathrm{a}}(0.033)$ & $0.804^{\mathrm{b}}(0.063)$ & BDL & $0.803^{\mathrm{b}}(0.024)$ & (X) & $\mathrm{X}$ & na & 12.77 & 2,17 & $<0.0004$ \\
\hline $18: 4 n-3$ & $\mathrm{E}, \mathrm{D}$ & $0.784^{\mathrm{C}}(0.016)$ & $0.420^{\mathrm{a}}(0.019)$ & $1.514^{\mathrm{d}}\left(0.073^{1}\right)$ & $0.541^{\mathrm{b}}(0.044)$ & $\mathrm{X}$ & $\mathrm{X}$ & $\mathrm{X}$ & 75.26 & 3,18 & $<0.0001$ \\
\hline $18: 4 n-1$ & $\mathrm{E}$ & $1.598(0.155)$ & $1.691(0.186)$ & BDL & $1.175(0.040)$ & & & na & & & 0.1384 \\
\hline $20: 1 n-11$ & $\mathrm{E}, \mathrm{D}$ & $2.352^{\mathrm{b}}(0.088)$ & $1.651^{\mathrm{a}}(0.074)$ & $1.742^{\mathrm{a}}(0.048)$ & $1.871^{\mathrm{a}}(0.079)$ & $(\mathrm{X})$ & $\mathrm{X}$ & & 11.52 & 3,18 & 0.0002 \\
\hline $20: 1 n-9$ & $\mathrm{E}, \mathrm{D}$ & $3.149^{\mathrm{c}}(0.159)$ & $1.435^{\mathrm{b}}(0.109)$ & $2.090^{\mathrm{c}}\left(0.093^{1}\right)$ & $0.789^{\mathrm{a}}(0.030)$ & $\mathrm{X}$ & $\mathrm{X}$ & $\mathrm{X}$ & 50.25 & 3,18 & $<0.0001$ \\
\hline $20: 1 n-7$ & $\mathrm{E}, \mathrm{D}$ & $0.905^{b}\left(0.047^{1}\right)$ & $1.086^{c}\left(0.042^{1}\right)$ & $1.126^{\mathrm{c}}\left(0.025^{1}\right)$ & $0.625^{\mathrm{a}}(0.017)$ & $\mathrm{X}$ & $\mathrm{X}$ & & 24.68 & 3,18 & $<0.0001$ \\
\hline $20: 2 n-6$ & $\mathrm{E}, \mathrm{D}$ & $1.047^{\mathrm{b}}\left(0.028^{1}\right)$ & $1.144^{\mathrm{b}}(0.033)$ & $2.258^{\mathrm{c}}(0.057)$ & $0.881^{\mathrm{a}}\left(0.042^{1}\right)$ & $\mathrm{X}$ & & $\mathrm{X}$ & 59.64 & 3,18 & $<0.0001$ \\
\hline $20: 3 n-6$ & $\mathrm{E}, \mathrm{D}$ & $1.127^{\mathrm{b}}(0.012)$ & $1.451^{\mathrm{C}}(0.058)$ & $2.826^{\mathrm{d}}(0.035)$ & $0.911^{\mathrm{a}}(0.016)$ & $\mathrm{X}$ & $X$ & $\mathrm{X}$ & 70.32 & 3,18 & $<0.0001$ \\
\hline $20: 4 n-6$ & $\mathrm{E}, \mathrm{D}$ & $0.652^{\mathrm{a}}(0.024)$ & $0.860^{\mathrm{b}}(0.022)$ & $1.664^{\mathrm{c}}\left(0.092^{1}\right)$ & $0.932^{\mathrm{b}}\left(0.022^{1}\right)$ & (X) & $\mathrm{X}$ & $\mathrm{X}$ & 74.38 & 3,18 & $<0.0001$ \\
\hline $20: 3 n-3$ & $\mathrm{E}, \mathrm{D}$ & $1.106^{\mathrm{b}}(0.020)$ & $0.844^{\mathrm{a}}(0.034)$ & BDL & $0.742^{\mathrm{a}}(0.023)$ & (X) & $\mathrm{X}$ & na & 25.36 & 2,16 & $<0.0001$ \\
\hline $20: 4 n-3$ & $\mathrm{E}, \mathrm{D}$ & $1.500^{\mathrm{b}}(0.070)$ & $1.466^{\mathrm{b}}(0.054)$ & $2.453^{\mathrm{c}}(0.001)$ & $1.034^{\mathrm{a}}\left(0.059^{1}\right)$ & $\mathrm{X}$ & & $\mathrm{X}$ & 26.77 & 3,18 & $<0.0001$ \\
\hline $20: 5 n-3$ & $\mathrm{E}, \mathrm{D}$ & $0.482^{\mathrm{a}, \mathrm{b}}(0.036)$ & $0.378^{\mathrm{a}}(0.026)$ & $0.922^{\mathrm{c}}\left(0.025^{1}\right)$ & $0.552^{\mathrm{b}}(0.039)$ & (X) & & $\mathrm{X}$ & 17.72 & 3,17 & $<0.0001$ \\
\hline $22: 1 n-11$ & $\mathrm{E}, \mathrm{D}$ & $1.324^{\mathrm{c}}(0.119)$ & $0.560^{\mathrm{b}}(0.054)$ & $0.663^{\mathrm{b}}\left(0.055^{1}\right)$ & $0.167^{a}(0.014)$ & $\mathrm{X}$ & $\mathrm{X}$ & & 46.30 & 3,18 & $<0.0001$ \\
\hline $22: 1 n-9$ & $\mathrm{E}, \mathrm{D}$ & $0.944^{\mathrm{b}}\left(0.080^{1}\right)$ & $0.845^{\mathrm{b}}(0.065)$ & $0.340^{\mathrm{a}}(0.021)$ & $0.246^{\mathrm{a}}(0.014)$ & $\mathrm{X}$ & & $\mathrm{X}$ & 43.59 & 3,18 & $<0.0001$ \\
\hline $22: 1 n-7$ & $\mathrm{E}, \mathrm{D}$ & $0.598^{\mathrm{b}}(0.057)$ & $0.525^{\mathrm{b}}(0.052)$ & BDL & $0.089^{\mathrm{a}}(0.008)$ & $\mathrm{X}$ & & na & 72.59 & 2,17 & $<0.0001$ \\
\hline $22: 2 n-6$ & $\mathrm{E}, \mathrm{D}$ & $0.913\left(0.094^{1}\right)$ & $1.000(0.002)$ & $\mathrm{BDL}$ & {$[0.000]$} & na & & na & & & 0.2649 \\
\hline $21: 5 n-3$ & $\mathrm{E}, \mathrm{D}$ & $0.968(0.008)$ & $0.805(0.041)$ & $\mathrm{BDL}$ & $0.884\left(0.054^{1}\right)$ & & & na & & & 0.0204 \\
\hline $22: 4 n-6$ & $\mathrm{E}, \mathrm{D}$ & $0.884^{\mathrm{a}}\left(0.058^{1}\right)$ & $1.320^{\mathrm{b}}(0.071)$ & $\mathrm{BDL}$ & $1.498^{\mathrm{b}}(0.068)$ & $(\mathrm{X})$ & $\mathrm{X}$ & na & 18.13 & 2,17 & $<0.0001$ \\
\hline $22: 5 n-6$ & $\mathrm{E}, \mathrm{D}$ & $1.122\left(0.049^{1}\right)$ & $1.182(0.057)$ & $\mathrm{BDL}$ & $0.988\left(0.030^{1}\right)$ & & & na & & & 0.1287 \\
\hline $22: 4 n-3$ & $\mathrm{E}, \mathrm{D}$ & $1.417^{\mathrm{b}}(0.044)$ & $0.972^{\mathrm{a}}\left(0.020^{1}\right)$ & BDL & $1.652^{\mathrm{c}}(0.057)$ & $\mathrm{X}$ & $\mathrm{X}$ & na & 108.8 & 2,17 & $<0.0001$ \\
\hline $22: 5 n-3$ & $\mathrm{E}$ & $2.879^{\mathrm{a}}(0.061)$ & $2.792^{\mathrm{a}}(0.107)$ & $4.813^{\mathrm{c}}\left(0.530^{1}\right)$ & $3.674^{\mathrm{b}}(0.102)$ & $\mathrm{X}$ & & $\mathrm{X}$ & 23.10 & 3,18 & $<0.0001$ \\
\hline $22: 6 n-3$ & $E, D$ & $0.911^{\mathrm{a}}(0.010)$ & $0.835^{\mathrm{a}}(0.023)$ & $1.567^{\mathrm{b}}\left(0.137^{1}\right)$ & $0.866^{\mathrm{a}}(0.024)$ & & & $\mathrm{X}$ & 39.57 & 3,18 & $<0.0001$ \\
\hline $12: 0$ & & $0.837(0.015)$ & $0.916\left(0.044^{1}\right)$ & $0.978\left(0.056^{1}\right)$ & $0.863(0.022)$ & & & & & & 0.3213 \\
\hline $13: 0$ & & $0.893^{\mathrm{c}}(0.029)$ & $1.001^{\mathrm{c}}(0.0002)$ & $0.832^{\mathrm{b}}\left(0.109^{1}\right)$ & $0.516^{\mathrm{a}}(0.038)$ & $\mathrm{X}$ & & $\mathrm{X}$ & 58.06 & 3,18 & $<0.0001$ \\
\hline$i-14: 0$ & & $0.937\left(0.027^{1}\right)$ & $1.001(0.0002)$ & $0.985\left(0.038^{1}\right)$ & $0.806\left(0.073^{1}\right)$ & & & & & & 0.0026 \\
\hline $14: 1 \mathrm{n}-9$ & & $1.305^{\mathrm{b}}(0.073)$ & $0.662^{\mathrm{a}}(0.042)$ & {$[0.000]$} & $0.823^{\mathrm{a}}\left(0.063^{1}\right)$ & $(\mathrm{X})$ & $\mathrm{X}$ & na & 28.87 & 2,17 & $<0.0001$ \\
\hline $14: 1 \mathrm{n}-7$ & & $1.359^{\mathrm{a}}(0.061)$ & $1.183^{\mathrm{a}}(0.046)$ & $1.479^{\mathrm{a}}\left(0.180^{1}\right)$ & $2.853^{\mathrm{b}}(0.153)$ & $\mathrm{X}$ & & & 52.27 & 3,18 & $<0.0001$ \\
\hline $14: 1 \mathrm{n}-5$ & & $1.509^{\mathrm{a}}(0.184)$ & $4.713^{\mathrm{b}}(0.353)$ & $2.908^{\mathrm{b}}\left(0.294^{1}\right)$ & $12.137^{\mathrm{c}}(0.619)$ & $\mathrm{X}$ & $\mathrm{X}$ & & 67.67 & 3,17 & $<0.0001$ \\
\hline $\mathrm{i}-15: 0$ & & $1.049^{\mathrm{c}}\left(0.020^{1}\right)$ & $0.780^{\mathrm{b}}(0.027)$ & $0.684^{b}\left(0.027^{1}\right)$ & $0.538^{\mathrm{a}}(0.026)$ & $\mathrm{X}$ & $\mathrm{X}$ & & 47.83 & 3,18 & $<0.0001$ \\
\hline ai- $15: 0$ & & $1.051\left(0.040^{1}\right)$ & $0.993\left(0.011^{1}\right)$ & BDL & $0.785\left(0.082^{1}\right)$ & & & na & & & 0.0021 \\
\hline $15: 0$ & & $0.972^{\mathrm{c}}(0.010)$ & $0.809^{\mathrm{b}}(0.014)$ & $1.053^{\mathrm{c}}\left(0.023^{1}\right)$ & $0.673^{\mathrm{a}}(0.006)$ & $\mathrm{X}$ & $\mathrm{X}$ & $\mathrm{X}$ & 79.40 & 3,18 & $<0.0001$ \\
\hline
\end{tabular}


Table 2 (continued)

\begin{tabular}{|c|c|c|c|c|c|c|c|c|c|c|}
\hline Subset & NFS HG & SSL HG & SSL EUL & PV HG & Family & Spp. & Prey & $F$ & df & $\mathrm{p}$ \\
\hline $15: 1 n-8$ & $1.112^{\mathrm{c}}(0.023)$ & $1.001^{\mathrm{b}}(0.0002)$ & BDL & $0.942^{\mathrm{a}}(0.001)$ & $\mathrm{X}$ & $\mathrm{X}$ & na & 34.32 & 2,17 & $<0.0001$ \\
\hline $15: 1 n-6$ & $1.239^{\mathrm{a}}\left(0.162^{1}\right)$ & $1.001^{\mathrm{a}}(0.0002)$ & BDL & $6.372^{\mathrm{b}}(0.352)$ & $\mathrm{X}$ & & na & 140.7 & 2,15 & $<0.0001$ \\
\hline $\mathrm{i}-16: 0$ & $0.921(0.014)$ & $0.884(0.029)$ & $1.147\left(0.065^{1}\right)$ & $0.812(0.024)$ & & & & & & 0.001 \\
\hline $16: 1 n-11$ & $1.005^{\mathrm{b}}\left(0.034^{1}\right)$ & $1.038^{\mathrm{b}}\left(0.045^{1}\right)$ & $0.440^{\mathrm{a}}(0.020)$ & $1.898^{\mathrm{c}}(0.077)$ & $\mathrm{X}$ & & $\mathrm{X}$ & 79.38 & 3,18 & $<0.0001$ \\
\hline $16: 1 n-9$ & $1.767^{\mathrm{b}}(0.059)$ & $1.976^{\mathrm{b}}(0.077)$ & $1.216^{\mathrm{a}}\left(0.044^{1}\right)$ & $3.506^{\mathrm{c}}(0.077)$ & $\mathrm{X}$ & & $\mathrm{X}$ & 59.74 & 3,18 & $<0.0001$ \\
\hline $7 \mathrm{Me} 16.0$ & BDL & $0.802(0.037)$ & $1.176\left(0.035^{1}\right)$ & $0.976\left(0.024^{1}\right)$ & & na & & & & 0.0016 \\
\hline $16: 1 n-5$ & $0.871^{\mathrm{a}}(0.018)$ & $0.939^{a}(0.020)$ & $0.830^{\mathrm{a}}\left(0.020^{1}\right)$ & $1.550^{\mathrm{b}}(0.094)$ & $\mathrm{X}$ & & & 59.26 & 3,17 & $<0.0001$ \\
\hline $\mathrm{i}-17: 0$ & $0.849^{\mathrm{b}}(0.009)$ & $0.852^{\mathrm{b}}(0.023)$ & $0.660^{\mathrm{a}}(0.014)$ & $0.864^{\mathrm{b}}(0.007)$ & & & $\mathrm{X}$ & 12.26 & 3,17 & 0.0002 \\
\hline $17: 1$ & $1.032^{\mathrm{a}}\left(0.047^{1}\right)$ & $1.132^{\mathrm{a}}(0.045)$ & [0.000] & $1.618^{\mathrm{b}}(0.022)$ & $\mathrm{X}$ & & na & 22.43 & 2,17 & $<0.0001$ \\
\hline $18: 1 n-13$ & $1.497\left(0.215^{1}\right)$ & $1.214\left(0.135^{1}\right)$ & BDL & $2.530(0.061)$ & & & na & & & 0.0009 \\
\hline $18: 1 n-11$ & $2.277^{a}(0.127)$ & $4.219^{\mathrm{b}}(0.558)$ & $3.351^{\mathrm{a}, \mathrm{b}}(0.066)$ & $7.194^{\mathrm{C}}(0.318)$ & $\mathrm{X}$ & $\mathrm{X}$ & & 10.95 & 3,18 & 0.0003 \\
\hline $18: 1 n-5$ & $0.935^{\mathrm{b}}(0.007)$ & $0.872^{\mathrm{b}}(0.018)$ & {$[0.000]$} & $0.763^{\mathrm{a}}(0.015)$ & $\mathrm{X}$ & & na & 19.36 & 2,17 & $<0.0001$ \\
\hline $18: 2 \mathrm{~d} 5,11$ & $1.257^{\mathrm{C}}(0.081)$ & $0.925^{\mathrm{a}}(0.014)$ & $0.946^{a, b}\left(0.101^{1}\right)$ & $1.120^{\mathrm{b}, \mathrm{c}}\left(0.053^{1}\right)$ & 1) $(\mathrm{X})$ & $\mathrm{X}$ & & 11.67 & 3,18 & 0.0002 \\
\hline $18: 2 n-7$ & $1.119^{\mathrm{b}}(0.039)$ & $1.242^{\mathrm{b}}(0.033)$ & $0.746^{\mathrm{a}}\left(0.080^{1}\right)$ & $1.690^{\mathrm{C}}(0.014)$ & $\mathrm{X}$ & & $\mathrm{X}$ & 45.27 & 3,18 & $<0.0001$ \\
\hline $20: 0$ & $0.759(0.059)$ & $0.761(0.056)$ & $1.098\left(0.012^{1}\right)$ & $0.471(0.023)$ & & & & & & 0.0014 \\
\hline $20: 2 n-9$ & {$[0.000]$} & BDL & BDL & $0.476(0.010)$ & na & na & na & na & na & na \\
\hline
\end{tabular}

tistical interpretation to the 41 FAs comprising the 'extended dietary fatty acid' (EDFA) subset, as described by Iverson et al. (2004) (identified in Table 1).

To investigate the effect of using different CCs to predict diet using QFASA, 8 subadult SSL (the same individuals used in the calibration trials) were fed a consistent diet of Pacific herring (64.3\%), eulachon $(14.3 \%)$, California market squid (Loligo opalescens, $14.3 \%$ ) and rockfish (Pacific ocean perch Sebastes alutus, $7.1 \%$ ) for a total of $112 \mathrm{~d}$. Full depth blubber biopsies were collected on Day 113 and analysed for FA profile following the procedures described above. Diet estimates were predicted using QFASA R-code provided by the Department of Biology, Dalhousie University (version 2007), and the methodology described by Iverson et al. (2004). QFASA runs were undertaken using the 'dietary' FA subset and applying the herring- and eulachon-derived CCs previously described to each whole blubber sample. The 'dietary' FA subset is similar to the EDFA subset, except for the exclusion of 8 FAs (see Table 2), which is reported to increase diet predictability in some cases (Nordstrom et al. 2008, Iverson 2009). Thirteen species of potential prey were included in each of the model runs, including the prey FA signatures of those 4 prey species fed during the feeding study, plus 4 additional species of forage fish (capelin, pilchard, sandlance and surf smelt) and 5 other fish species (walleye pollock, Atka mackerel, coho salmon, Sockeye salmon and pink salmon) that were not fed. Mean $( \pm \mathrm{SD})$ diet predictions were generated for the 8 SSL for each prey species fed plus the combined contribution from these 2 prey groupings (other forage fish, other fish).

\section{RESULTS}

Not all of the 66 FAs were present in levels above detection thresholds (minimal concentrations that can be reliably detected as determined a priori by each laboratory) in all prey samples. For example, each profile for herring contained between 63 and 65 FAs depending on the lab analysis/fish batch (Table 1). In comparison, analysis of eulachon yielded 49 FAs above detection limits for the complete FA profile and 29 for the EDFA profile. It is impossible to determine the degree to which these differences (particularly between herring batches) were due to true biochemical differences or attributable to laboratory differences. For the goals of our study, it was critical that we used matched predator/prey profiles rather than creating a composite prey profile.

However, detectable levels were common to at least 2 species/prey combinations for 40 of 41 FAs contributing to the EDFA profile (65 of 66 FAs in the complete dataset). The mean CCs derived for each of the predator/prey combinations are listed in Table 2.

\section{Variation in FA signatures between predator and prey}

CCs that differ from 1.0 indicate preferential accumulation, mobilization or transformation of FAs following consumption. There were substantial differences in the \%FA for a given FA between the predators and prey as demonstrated by the number of CCs that significantly differed from 1.0 (i.e. where the $\%$ FA in the predator and prey would be statistically equal; Fig. 1, Table 1). 


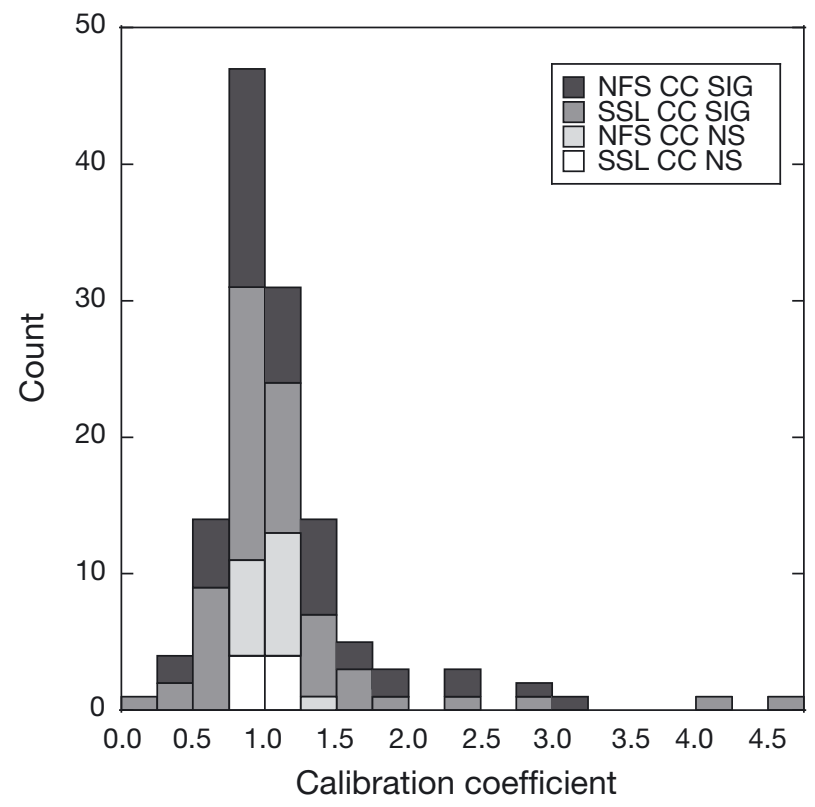

Fig. 1. Frequency distribution of mean calibration coefficients (CC) for fatty acids (FAs) from both northern fur seals $\left(N_{F S} ;=62\right)$ and Steller sea lions (SSL; $\left.\mathrm{n}=64\right)$ consuming herring. Each data point represents a $\mathrm{CC}$ for an individual FA for either species of predator. Coefficients that are significantly different from 1.0 ('CC SIG', i.e. significant differences in representation between predator and prey) for each species are designated by darker shading, while those not significantly different from 1.0 are given lighter shading ('CC NS')

For the 41 FAs comprising the EDFA subset, the mean CCs for SSL, PV and NFS consuming herring were statistically different from 1.0 in $93 \%(37 / 40)$, $79 \%(31 / 39)$ and $74 \%(29 / 39)$ of cases, respectively. For SSL consuming eulachon, $36 \%(10 / 28)$ of the CCs were different from 1.0. The results were very similar for the complete FA data set $(89,80,73$ and $51 \%$, respectively; Fig. 1).

These differences were substantial. For example, for SSL eating herring, 4 CCs were $>2.0$ and 3 were $<0.5$, with all but 2 of the FAs belonging to the EDFA subset. Similarly, for NFS there were 4 CCs $>2.0$ and $2<0.5$, with all but $1 \mathrm{FA}$ being members of the EDFA subset (Table 2).

There was variation associated with the CCs for each FA. We hypothesized that some of the variation would be associated with small \%FA representation in either the blubber or herring (partly measurement error, partly the effects of natural scope of variation). We found in both SSL and NFS that the highest SD for an individual CC was associated with the lowest \%FA values in both the blubber and herring samples. In addition, there was a relationship between CCs and variance, whereby increasing larger CCs were significantly associated with larger SDs (overall $\mathrm{p}<$ $0.001, \mathrm{r}^{2}=0.45$ ).

\section{Classification of differences across phylogeny and prey type}

Of the 41 FAs comprising the EDFA subset, $41 \%$ $(16 / 39$; due to lack of data it was not possible to make a family-wise comparison for 2 FAs) differed by family (Table 2). That is, the CCs for PV eating herring were significantly different from both those for NFS and SSL also consuming herring. An additional $28 \%$ (11/39) differed between PV and either NFS or SSL consuming herring, but not both. For the complete set of 66 FAs, $48 \%$ (30 of 63 potential comparisons) CCs differed by family, and an additional $21 \%$ (13/63) of the CCs for PV eating herring differed from either SSL or NFS.

We also analyzed the CCs to determine which differed by predator species, i.e. differed between NFS and SSL both eating herring. Within the EDFA subset, $58 \%(22 / 38)$ differed by predator species (it was not possible to make similar comparisons for $3 \mathrm{FAs}$ ). In the complete FA dataset, we found a statistical difference in $48 \%$ (29/61) of CCs (Fig. 2). The largest differences were observed in the FAs 16:4n-1, 17:0, 20:1n-9 and 22:1n-11, where the ratio between CCs for NFS and SSL was $>2.0$ and in 14:1n-5, where the ratio was $<0.5$. All but the last are members of the EDFA subset.

Finally, we calculated the number of FAs that differed by prey consumed by comparing CCs of SSL eating herring or eulachon. In total, $64 \%(18 / 28)$ of $\mathrm{CCs}$ in the EDFA dataset differed by prey type. Unfortunately, for $32 \%$ of FAs (13/41) it was not possible to make a comparison as we were unable to calculate a robust CC for SSL consuming eulachon for these FAs. Similarly, in the complete FA dataset, there was a statistical difference in the CCs of $53 \%$ (24/45) of comparable FAs (Table 2). It was impossible to make such a comparison in $32 \%(21 / 66)$ of FAs due to lack of matching CCs.

\section{Effect of different CCs on prey predictions}

The results of our initial trials predicting a constant, multi-species diet in SSL demonstrated significant differences depending on whether the herring- or eulachon-derived CCs were used (Fig. 3). Regardless of the CCs used, there was generally mediocre 


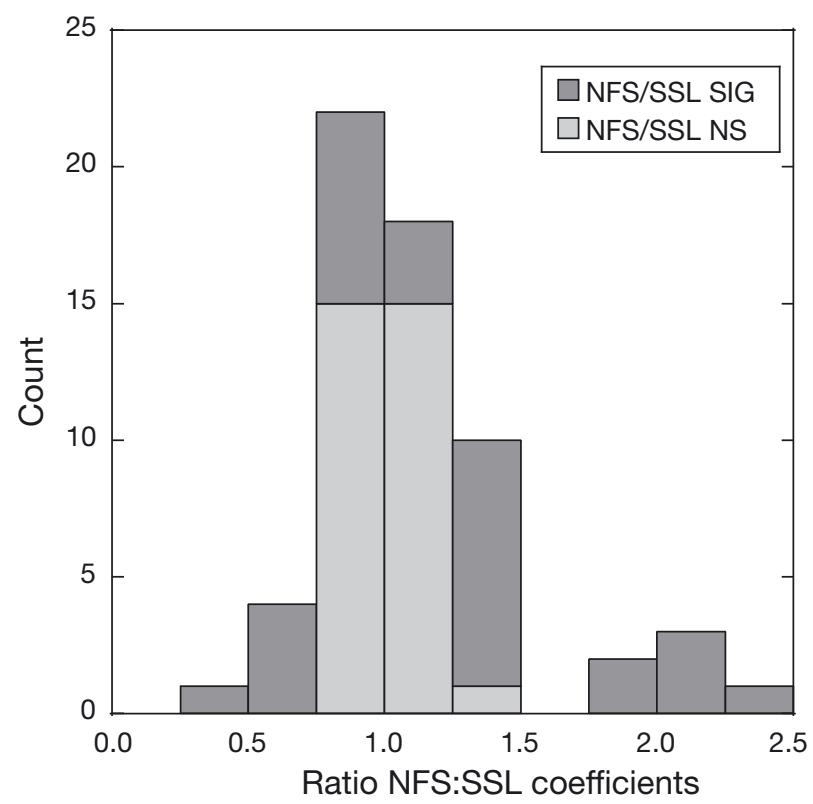

Fig. 2. Frequency distribution of the relationship between calibration coefficients (CC) for fatty acids (FAs) from northern fur seals (NFS) and Steller sea lions (SSL) consuming herring. Each of the 61 data points represents a CC for an individual FA common to both predator species (listed in Table 2). Values are calculated as the ratio of CCs between NFS and SSL. Darker shading distinguishes results from FAs where CCs from SSL and NFS were significantly different from each other (NFS/SSL SIG) while lighter shading denotes non-significant differences (NFS/SSL NS)

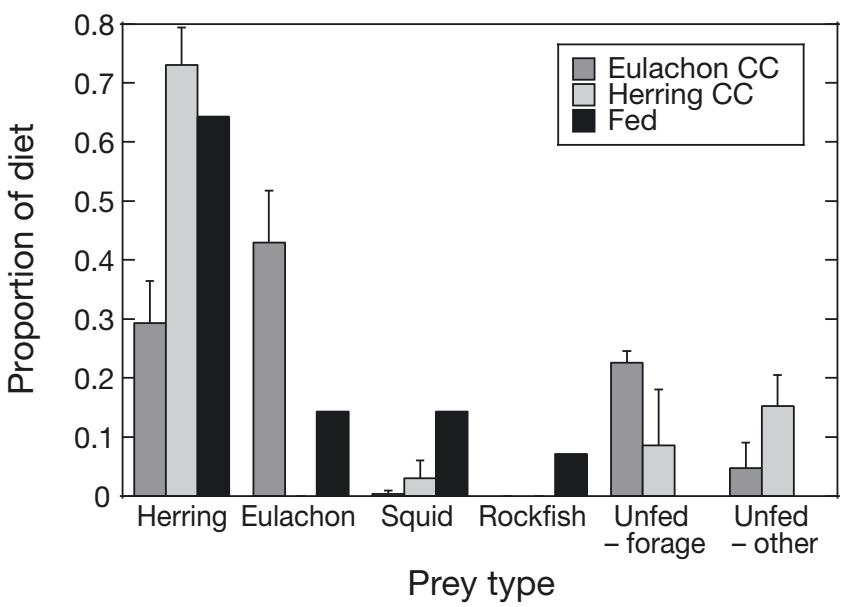

Fig. 3. Results of a QFASA prediction of diet (mean + $1 \mathrm{SD}$ ) comparing results from using either a eulachon- or herringderived calibration coefficient (CC) with actual prey fed (black bars). Data are from a study where 8 Steller sea lions were consuming a constant mixed diet of 4 prey items (herring, eulachon, squid, and rockfish) for $112 \mathrm{~d}$. Also indicated are the 'misclassified' prey that were not fed, categorized generally into 'forage fish' and 'other' agreement between predicted and actual diet, with maximum overlap averaging $67.4 \%$ (herring $\mathrm{CCs}$ ) and $44.1 \%$ (eulachon CCs). As predicted, the herring CCs most accurately predicted the amount of herring fed, but failed to detect the eulachon (while the eulachon CCs considerably over-predicted the amount of eulachon fed). The herring CCs did a better (although still inadequate) job of identifying squid in the diet, and neither set of CCs detected the rockfish. Both CCs predicted substantial (23 to $27 \%$ ) amounts of prey that were never fed (Fig. 3).

\section{DISCUSSION}

Accurate determination of diet is critical for understanding the ecological role of predators such as pinnipeds. The effect of pinnipeds on their prey or the effect of changes in potential prey on pinniped populations encompasses the vast majority of management and conservation issues related to this group of mammals (e.g. Wickens et al. 1992, Boyd 2002, Corkeron 2009, Mangel 2010). Among the shortfalls of many measures of diet determination is the inherent constraint that they are only able to relate the immediate ( 24 to $48 \mathrm{~h}$ ) dietary history of an individual (Tollit et al. 2010). QFASA is a tool specifically designed to estimate the long-term diet of individuals (Iverson et al. 2004). The difficulties inherent in describing the diet of pinnipeds and other marine mammals make QFASA a tool worthy of substantial investment and testing.

However, like all tools, QFASA is designed to be used in a specific manner and will result in questionable or erroneous results if misused. Further, it is a technique that should still be considered to be under development, both in its internal workings and its implementation. Probably the most common misuse of QFASA lies in the misapplication of inappropriate CCs. To date, few CCs are available for phocid pinnipeds (i.e. PV, grey seals, harp seals) and none for otariids. This paucity of data is due to the considerable logistical difficulties in obtaining samples from individuals that have consumed a known diet for a period sufficient (e.g. many months) to ensure that the resulting lipid storage body wholly reflects that prey source.

\section{Importance of $\mathrm{CCs}$}

Given the difficulties in obtaining CCs, it is critical to ask the fundamental question of 'how important 
are they?' Specifically, are CCs required to adjust the FA profile of the predator to better reflect that of its prey? In our study, the proportional representation of the vast majority of the 41 FAs in the EDFA subset were significantly different between predator and prey (similar to the results for the complete FA dataset). That is, the majority of CCs were significantly different from unity, particularly in individuals consuming herring (the greater proportion of CCs that were not significantly different from 1.0 for SSL consuming eulachon is considered partly the result of smaller samples sizes). The number of FAs with a CC significantly different than 1.0 is much higher than for those reported for 2 wk old grey seal pups (values often used in QFASA analyses, e.g. Beck et al. 2007, Tucker et al. 2009). As noted by Nordstrom et al. (2008), grey seal pup CCs were based on an exceedingly high (i.e. $60 \%$ ) fat milk, which probably suppressed FA biosynthesis completely (Iverson et al. 2004), thereby contributing to the prevalence of CCs approaching unity.

The observed variation from unity (1.0) in our study highlights that CCs are required in the QFASA process to take into account differential synthesis and metabolism. This confirms that 'perhaps the most important issue when using FA quantitatively is accounting for predator metabolism' (Iverson 2009, p. 298). CCs $>1.0$ result from situations where the $\% \mathrm{FA}$ of an FA in the predator is greater than in the prey, and are presumed to result from its bioaccumulation or biotransformation from other FAs. The latter is typified by the endogenous synthesis of shortchain saturated FAs and monounsaturated FAs. Very high CCs might mean that these FAs are not particularly useful for QFASA, as they may be too subject to biological processes - and generally associated with high variance - to be representative of dietary FAs. Mathematically, very high CCs will result in substantial 'adjustments' that might result in unacceptably large errors. In both our NFS and SSL samples, higher CCs were indeed associated with higher variance. This observation is particularly important given the recent interest in using bootstrapping resampling techniques for dietary FA investigations (Stewart \& Field 2011, Thiemann et al. 2011).

Essential FAs represent a special case, as the predator cannot synthesize them. However, this does not mean that they are necessarily present in the predator in the same ratio as in the prey. The CCs for the essential FA linoleic acid (18:2n-6) for all predator/ prey combinations were significantly greater than 1.0, and there were also significant differences attributable to predator and prey type (Table 2). The same trends were generally true (except for PV) for the other essential FA, alpha-linolenic acid (18:3n-3).

Conversely, CCs $<1.0$ represent the case where FAs are either not integrated into the blubber layer or are preferentially broken down or transformed. They do not appear to differ as far from unity as CCs $>1.0$, but only because they are mathematically limited to values between 1 and 0 . For this reason, there is cause to suspect that a CC close to 0 may be subject to more potential bias than a CC that is a similar distance above unity.

FAs that have a calculated CC of 0 (i.e. where no detectable quantities are measured in blubber, regardless of representation in prey, such as 14:1n-9, $17: 1,18: 1 \mathrm{n}-5$ for SSL consuming eulachon) are a particular case, and obviously cannot be used for dietary modelling. Similarly, there are instances where there are detectable FA levels in the predator but not the prey, such that CCs are not calculable. While these latter are usually (but not exclusively; see Table 1) found only on a fish-to-fish basis, it does raise concerns about calculating a mean CC based only on the subset of fish samples where the FA is detectable. The implications of this circumstance are further addressed below. It also raises the issue of reliable minimal estimates of lipid representation. This is important given the not surprising observation that very high uncertainty in CCs in our study was associated with FAs in very low abundance in either predator or prey. Meynier et al. (2010) similarly found that removal of FAs with relatively low mass improved the results of their QFASA diet predictions in New Zealand (NZ) fur seals Arctocephalus forsteri. Given that the choice of the FA set used can significantly affect diet estimates, subset selection should be thoroughly tested by the investigator.

\section{Effect of predator species on CCs}

Given the inherent logistical and financial difficulties, it is not surprising that, as the use of QFASA increases, there is a tendency to apply CCs derived for one species to other study species. Some studies are able to apply CCs derived from apparently closely related species (e.g. SSL for NZ sea lions; Meynier et al. 2010), while others average a number of published CCs in an attempt to 'at least partially account for any uncertainty in this parameter' (Tucker et al. 2009, p. 300). Others have had to make use of CCs derived from less clearly related species, such as substituting mink for polar bears (Thiemann et al. 2007). This liberal use of CCs potentially represents a 
dangerous application of a set of correction factors that may be highly species-specific. However, given that it is practically impossible for a host of logistical and financial reasons to derive a unique set of CCs for each predator species under investigation, the question emerges regarding how closely related 2 species need to be in order to confidently apply CCs from one to the other.

It has been demonstrated that cetaceans and otariids consuming similar prey have significantly different FA profiles, suggesting phylogenetic differences in lipid utilization (Grahl-Nielsen et al. 2010). It is not unrealistic to hypothesize that CCs might differ between otariid and phocid seals, as the extent of the blubber layers and potentially the underlying lipid dynamics (related to the degree of reliance as a seasonal energy reserve) differ between them. In our study, a substantial proportion $(\sim 50 \%)$ of the CCs for an individual FA were significantly different between our model phocid (PV) and both of our otariid species (NFS and SSL) while consuming identical prey type (herring). This observation held whether discussing the complete FA set or the EDFA subset. An additional $20 \%$ of FAs differed between the PV and one of the otariid species. In the end, our study represents the first CCs specifically derived for otariid species. These 2 sets of CCs should improve model predictions for researchers determining the diet of otariid species through QFASA.

However, these results raise the additional question of whether family-specific sets of CCs are sufficient, or whether there are also significant differences in CCs between predators at the species level. In our study, the CCs for half of the FAs differed between NFS and SSL when both were consuming herring. Similarly, Nordstrom et al. (2008) found significant differences in the CCs of some FAs derived from PV consuming herring, and those previously reported for other phocids by Iverson et al. (2004) consuming similar prey. Nordstrom et al. (2008) were unable to determine whether these differences were due to species effects or to other experimental differences (age, growth, lipid content of prey) including the considerably longer monotypic feeding period that they employed.

However, it is important to acknowledge that statistical differences do not necessarily result in significantly different diet predictions. The effect of differing CCs will likely be greatest for those FAs with the greatest discrepancy in CCs. We can calculate this as the ratio between the CCs for NFS and SSL for each FA (Fig. 2). In our study, the CCs for all but 6 FAs fell between the ratios of 0.5 and 2.0 (with no discernible trends related to FA size or quantity). While this might seem reasonable, it should be noted that the difference in these CCs represents a potential 100\% difference in the effective CCs between these 2 predator species.

\section{Effect of prey-specific differences in CCs}

Perhaps the result of greatest concern arising from the present study is the difference in CCs reported for SSL consuming long-term diets of herring and eulachon. We found a statistical difference in just over half of the FAs, either when comparing the complete data set or just the more diet-related EDFA subset. It is notable that it was impossible to make prey comparisons between herring and eulachon for about one-third of the FAs, primarily because we were unable to calculate an appropriate CC for eulachon (Table 2). While this may be affected by differences in lower threshold detection criteria between laboratories, it more likely reflects true compositional and/or blubber integration differences between herring and eulachon.

To date, almost all published CCs have been undertaken with seals consuming herring, largely for practical reasons. Herring is a common diet component in captive pinnipeds as it is readily available and high in lipid content. Its characteristics of high lipid content and wide range of FA representation also make it a good prey candidate for developing CCs for QFASA. In contrast, it is evident from the process of deriving CCs from SSL consuming eulachon that a significant proportion of FAs were inadequately represented in both the prey and the predator. It must be reiterated that we only had 2 SSL consuming eulachon, so care must be taken in the interpretation of our results. However, statistically, the sample size for SSL consuming eulachon likely served to decrease the number of significant differences found in CCs between eulachon and herring. The tendency to use herring as a species for developing CCs might lead to certain biases, such as an increased ability to detect herring in the diet (see Budge et al. 2012).

Of course, the 'acid test' of the effect of predatorspecific CCs is to actually run QFASA with the different sets of CCs and quantify the differences in diet prediction. The CCs developed by Nordstrom et al. (2008) from PV consuming herring did a better subsequent job of predicting diets with a high proportion of herring than diets dominated by other prey species. This was also true for the example in our study 
of diet predictions using both eulachon- and herringderived CCs. However, the high overestimate of herring consumed may have also led to it vastly underestimating or even failing to detect other fed prey species, while still predicting substantial (24\%) amounts of prey that were never fed. Similarly, the eulachon-derived CCs overestimated the amount of eulachon actually consumed, while also misidentifying a high proportion $(27 \%)$ of unfed prey.

A similar exercise was undertaken by Meynier et al. (2010). They found that different sets of CCs (including several detailed in this paper) yielded significantly different dietary predictions for NZ sea lions. Their study showed that diet estimates switched from 1 highly dominant species (>70\% mass) to several major species and no representation from the initially dominant species, depending on the CCs used. Herring CCs from SSL were found to perform best, with the premise that this fish has an FA profile closer to the mean FA profile of NZ sea lion prey than eulachon. The potentially large effect of using different CCs on QFASA estimates was also highlighted by Hoberecht (2006).

Another set of biases might originate from the choice of FAs used in QFASA. Only a subset of FAs (such as the EDFA subset) is used in the QFASA process rather than the complete FA profile. The decision to use specific sets of FAs is partly determined by their ability to distinguish between prey types, but also by the requirement that they be present in analytically significant levels. Both of these characteristics will differ between prey species.

\section{Practical concerns with CCs in QFASA}

It is not our intention to disparage the QFASA method of diet quantification. Rather, we consider our study part of an ongoing process of development and refinement that should occur with all modelling tools, a process acknowledged by the originators of the QFASA methodology (Iverson 2009). Our study has raised some concrete operational questions regarding the development and use of CCs in the QFASA method for pinniped diet determination that need to be addressed either through further empirical studies or mathematical modelling.

(1) If there are statistical differences between CCs for predators consuming different prey, which set is most appropriate to use? Operationally, it might seem valid to use CCs derived from a major prey item, on the assumption that it might accurately estimate the largest component of the diet. However, even if this were an accurate assumption, it raises the spectre of a potential circular argument, i.e. that one needs to already know the diet to apply the appropriate CCs.

(2) What CCs does one employ in the model for FAs that are unrepresented in either the prey or the predator? In our study it was not possible to calculate a CC for $31 \%$ of FAs of the EDFA subset for eulachon. Operationally, it might be suggested to use a value of '1.0', but this introduces an unquantified bias. For example, in our study, in most of the cases (10/13) where a CC could not be calculated for eulachon, the corresponding $\mathrm{CC}$ for herring significantly differed from 1.0 .

(3) The CCs for some FAs differ between prey species more than others. It might be suggested that the FA subset used can be trimmed to eliminate such prey-centric discrepancies. However, can QFASA still work by removing the more radically different FAs, especially as these may be particularly useful for differentiating prey? Further, does this mean that in order to implement such a modification to QFASA it may but necessary to do calibration studies for all significant prey species?

(4) If biotransformation and differential utilization are rate processes (like many physiological systems), then $\mathrm{CCs}$ - which represent a proportional change - may not be constant for different prey species with different quantities of FAs. This suggests that the current method is not the best for deriving correction factors. Further research needs to be undertaken to explore alternative methods to quantify these underlying physiological processes. Turnover of specific FAs can be calculated by turnover of labelled radioisotopes (Budge et al. 2004, Cooper et al. 2006) or by sequential sampling of lipids over the course of known dietary shifts.

(5) Finally, further research with animals under controlled feeding conditions needs to be conducted on how CCs (regardless of the methodology used to derive them) are affected by age, reproductive status, growth and energetic status. While these processes are all acknowledged to have an effect on lipid dynamics (e.g. Castellini et al. 1987, Kirsch et al. 2000, Wheatley et al. 2008), the ability to account for these processes in the QFASA model needs to be quantified through controlled studies. However, given the tremendous time and cost required for such studies, careful consideration should be given as to the greatest priorities for investigating these potential factors in future studies.

While FAs only provide indirect evidence of diet (compared to discernible hard remains, for example), potentially useful estimates of long-term diet can be 
collected directly from individual marine mammals. However, QFASA is relatively new and is still being refined. Ultimately, no single technique will provide all the answers, and researchers should aim to use as many sources of information as possible when weighing the evidence for marine mammal dietary compositions, especially if the species under study is a generalist consuming many different taxa. As noted by Iverson (2009, p. 301), 'QFASA must be used with due diligence and investigators should understand that there are many issues that remain to be resolved, or need to be further investigated, to improve its reliability.'

These warnings should not be taken lightly. We understand that, given the dominant theme of potential conflict between marine mammal populations and fisheries, resource managers require information on diet to aid policy makers. However, given the results of this study, we believe QFASA alone should not yet be used as a quantified descriptor of diet for any stock management decisions relating to pinnipeds, particularly if there are potentially severe (or lethal) consequences of such policies.

Acknowledgements. We thank the research and husbandry staff of the Marine Mammal Energetics and Nutrition Laboratory for their assistance, and the Vancouver Aquarium for additional support. L. Rea and 4 anonymous reviewers provided welcome feedback on an earlier draft of this manuscript. Research funding was provided to the North Pacific Universities Marine Mammal Research Consortium by the North Pacific Marine Science Foundation and the National Oceanic and Atmospheric Administration

\section{LITERATURE CITED}

Andersen SM, Lydersen C, Grahl-Nielsen O, Kovacs KM (2004) Autumn diet of harbour seals (Phoca vitulina) at Prins Karls Forland, Svalbard, assessed via scat and fatty-acid analyses. Can J Zool 82:1230-1245

- Bearzi G, Agazzi S, Gonzalvo J, Bonizzoni S, Costa M, Petroselli A (2010) Biomass removal by dolphins and fisheries in a Mediterranean Sea coastal area: Do dolphins have an ecological impact on fisheries? Aquat Conserv 20:549-559

Beck CA, Iverson SJ, Bowen WD, Blanchard W (2007) Sex differences in grey seal diet reflect seasonal variation in foraging behaviour and reproductive expenditure: evidence from quantitative fatty acid signature analysis. J Anim Ecol 76:490-502

> Bowen WD (1997) Role of marine mammals in aquatic ecosystems. Mar Ecol Prog Ser 158:267-274

Boyd IL (2002) Integrated environment-prey-predator interactions off South Georgia: implications for management of fisheries. Aquat Conserv 12:119-126

Budge SM, Iverson SJ, Bowen WD, Ackman RG (2002) Among- and within-species variability in fatty acid signatures of marine fish and invertebrates on the Scotian Shelf, Georges Bank, and southern Gulf of St. Lawrence.
Can J Fish Aquat Sci 59:886-898

Budge SM, Cooper MH, Iverson SJ (2004) Demonstration of the deposition and modification of dietary fatty acids in pinniped blubber using radiolabelled precursors. Physiol Biochem Zool 77:682-687

Budge SM, Penney SN, Lall SP (2012) Estimating diets of Atlantic salmon (Salmo salar) using fatty acid signature analyses; validation with controlled feeding studies. Can J Fish Aquat Sci 69:1033-1046

> Castellini MA, Costa DP, Huntley AC (1987) Fatty acid metabolism in fasting elephant seal pups. J Comp Physiol B Biochem Syst Environ Physiol 157:445-449

Cooper MH, Iverson SJ, Heras H (2005) Dynamics of blood chylomicron fatty acids in a marine carnivore: implications for lipid metabolism and quantitative estimation of predator diets. J Comp Physiol B Biochem Syst Environ Physiol 175:133-145

Cooper MH, Iverson SJ, Rouvinen-Watt K (2006) Metabolism of dietary cetoleic acid (22: 1n-11) in mink (Mustela vison) and gray seals (Halichoerus grypus) studied using radiolabeled fatty acids. Physiol Biochem Zool 79:820-829

> Corkeron PJ (2009) Marine mammals' influence on ecosystem processes affecting fisheries in the Barents Sea is trivial. Biol Lett 5:204-206

Dalsgaard J, St. John M, Kattner G, Müller-Navarra D, Hagen W (2003) Fatty acid trophic markers in the pelagic marine environment. Adv Mar Biol 46:225-340

> DeMaster D, Fowler C, Perry S, Richlen M (2001) Predation and competition: the impact of fisheries on marinemammal populations over the next one hundred years. J Mammal 82:641-651

> Gibbons RD (1995) Some statistical and conceptual issues in the detection of low-level environmental pollutants. Environ Ecol Stat 2:125-145

Grahl-Nielsen O, Andersen M, Derocher AE, Lydersen C, Wiig Ø, Kovacs KM (2003) Fatty acid composition of the adipose tissue of polar bears and of their prey: ringed seals, bearded seals and harp seals. Mar Ecol Prog Ser 265:275-282

> Grahl-Nielsen O, Krakstad JO, Nøttestad L, Axelsen BE (2010) Dusky dolphins Lagenorhynchus obscurus and Cape fur seals Arctocephalus pusillus pusillus: fatty acid composition of their blubber and prey species. Afr J Mar Sci 32:187-196

Hoberecht LK (2006) Investigating the use of blubber fatty acids to detect Steller sea lion (Eumetopias jubatus) foraging on ephemeral high-quality prey. PhD disertation, University of Washington, Seattle, WA

> Hobson KA (1999) Tracing origins and migration of wildlife using stable isotopes: a review. Oecologia 120:314-326

Iverson SJ (1993) Milk secretion in marine mammals in relation to foraging: Can milk fatty acids predict diet? Symp Zool Soc Lond 66:263-291

Iverson SJ (2009) Tracing aquatic food webs using fatty acids: from qualitative indicators to quantitative determination. In: Arts MT, Brett MT, Kainz MJ (eds) Lipids in aquatic ecosystems. Springer, p 281-308

Iverson SJ, Field C, Bowen WD, Blanchard W (2004) Quantitative fatty acid signature analysis: a new method of estimating predator diets. Ecol Monogr 74:211-235

Iverson S, Springer A, Kitaysky A (2007) Seabirds as indicators of food web structure and ecosystem variability: qualitative and quantitative diet analyses using fatty acids. Mar Ecol Prog Ser 352:235-244 
Kenney RD, Scott GP, Thompson TJ, Winn HE (1997) Estimates of prey consumption and trophic impacts of cetaceans in the USA Northeast continental shelf ecosystem. J Northwest Atl Fish Sci 22:155-171

Kennish JM, Beck CA, Rea LD, Iverson SJ and others (2006) A quality assurance and quality control program (QA/QC) for lipid extraction and gas chromatographic analysis for fatty acids (FA): an interlaboratory study. Marine Science in Alaska, Anchorage, AK

Kirsch PE, Iverson SJ, Bowen WD (2000) Effect of a low-fat diet on body composition and blubber fatty acids of captive juvenile harp seals (Phoca groenlandica). Physiol Biochem Zool 73:45-59

Mangel M (2010) Scientific inference and experiment in Ecosystem Based Fishery Management, with application to Steller sea lions in the Bering Sea and Western Gulf of Alaska. Mar Policy 34:836-843

Meynier L, Morel PCH, Chilvers BL, Mackenzie DDS, Duigan PJ (2010) Quantitative fatty acid signature analysis on New Zealand sea lions: model sensitivity and diet estimates. J Mammal 91:1484-1495

National Marine Fisheries Service (2007) Conservation plan for the Eastern Pacific stock of northern fur seal (Callorhinus ursinus). National Marine Fisheries Service, Juneau, AK

National Marine Fisheries Service (2008) Recovery plan for the Steller sea lion (Eumetopias jubatus). Revision. National Marine Fisheries Service, Silver Spring, MD

Nordstrom CA, Wilson LJ, Iverson SJ, Tollit DJ (2008) Evaluating quantitative fatty acid signature analysis (QFASA) using harbour seals Phoca vitulina richardsi in captive feeding studies. Mar Ecol Prog Ser 360:245-263

Editorial responsibility: Hans Heinrich Janssen, Oldendorf/Luhe, Germany
Stewart C, Field C (2011) Managing the essential zeros in quantitative fatty acid signature analysis. J Agric Biol Environ Stat 16:45-69

> Thiemann GW, Budge SM, Iverson SJ, Stirling I (2007) Unusual fatty acid biomarkers reveal age- and sexspecific foraging in polar bears (Ursus maritimus). Can J Zool 85:505-517

- Thiemann GW, Iverson SJ, Stirling I, Obbard ME (2011) Individual patterns of prey selection and dietary specialization in an Arctic marine carnivore. Oikos 120: 1469-1478

Tollit DJ, Pierce GJ, Hobson KA, Bowen WD, Iverson SJ (2010) Diet. In: Boyd IL, Bowen WD, Iverson SJ (eds) Marine mammal ecology and conservation: a handbook of techniques. Oxford University Press, Oxford, p 191-221

> Trites AW, Christensen V, Pauly D (1997) Competition between fisheries and marine mammals for prey and primary production in the Pacific Ocean. J Northwest Atl Fish Sci 22:173-187

Tucker S, Bowen WD, Iverson SJ, Blanchard W, Stenson GB (2009) Sources of variation in diets of harp and hooded seals estimated from quantitative fatty acid signature analysis (QFASA). Mar Ecol Prog Ser 384:287-302

> Wheatley KE, Nichols PD, Hindell MA, Harcourt RG, Bradshaw CJA (2008) Differential mobilization of blubber fatty acids in lactating Weddell seals: evidence for selective use. Physiol Biochem Zool 81:651-662

Wickens PA, Shelton PA, David JHM, Field JG, Oosthuizen WH, Roux JP, Starfield AM (1992) A fur seal simulation model to explore alternative management strategies. Can J Fish Aquat Sci 49:1396-1405

Submitted: November 16, 2011; Accepted: July 11, 2012 Proofs received from author(s): October 18, 2012 